\title{
Corrigendum: 9-PAHSA Improves Cardiovascular Complications by Promoting Autophagic Flux and Reducing Myocardial Hypertrophy in $\mathrm{Db} / \mathrm{Db}$ Mice
}

\section{OPEN ACCESS}

Approved by:

Frontiers Editorial Office,

Frontiers Media SA, Switzerland

${ }^{*}$ Correspondence:

Hou-Guang Zhou

zhouhouguang@huashan.org.cn Jing-Chun Guo

jingchunguo@shmu.edu.cn

Yan-Yan Huang

hyiwen94@hotmail.com

${ }^{\dagger}$ These authors have contributed equally to this work

Specialty section:

This article was submitted to Cardiovascular and Smooth Muscle

Pharmacology,

a section of the journal

Frontiers in Pharmacology

Received: 02 December 2021

Accepted: 08 December 2021

Published: 03 January 2022

Citation:

Wang $Y-M$, Mi S-L, Jin H, Guo Q-L,

$Y u Z-Y$, Wang $J-T$, Zhang $X-M$,

Zhang $Q$, Wang $N-N$, Huang $Y-Y$,

Zhou H-G and Guo J-C (2022)

Corrigendum: 9-PAHSA Improves

Cardiovascular Complications by

Promoting Autophagic Flux and Reducing Myocardial Hypertrophy in

$\mathrm{Db} / \mathrm{Db}$ Mice.

Front. Pharmacol. 12:827490.

doi: 10.3389/fphar.2021.827490
Yan-Mei Wang ${ }^{1 \dagger}$, Shou-Ling Mi ${ }^{2 \dagger}$, Hong Jin ${ }^{3 \dagger}$, Qi-Lin Guo ${ }^{1}$, Zhong-Yu Yu ${ }^{1}$, Jian-Tao Wang ${ }^{1}$, Xiao-Ming Zhang ${ }^{1}$, Qian Zhang ${ }^{1}$, Na-Na Wang ${ }^{1}$, Yan-Yan Huang ${ }^{1 *}$, Hou-Guang Zhou ${ }^{1 *}$ and Jing-Chun Guo ${ }^{1 *}$

${ }^{1}$ Department of Geriatrics of Huashan Hospital, National Clinical Research Center for Aging and Medicine, Department of Translational Neuroscience, Jing'an District Centre Hospital of Shanghai, State Key Laboratory of Medical Neurobiology and MOE Frontiers Center for Brain Science, Institutes of Brain Science, Fudan University, Shanghai, China, ${ }^{2}$ Department of Cardiology, Zhongshan Hospital, Fudan University, Shanghai, China, ${ }^{3}$ Shanghai Stomatological Hospital and Institutes of Biomedical Sciences, Fudan University, Shanghai, China

Keywords: diabetic cardiovascular complications, autophagy, 9-PAHSA, myocardial hypertrophy, vascular calcification

\section{A Corrigendum on}

9-PAHSA Improves Cardiovascular Complications by Promoting Autophagic Flux and Reducing Myocardial Hypertrophy in Db/Db Mice

by Wang, Y.-M., Mi, S.-L., Jin, H., Guo, Q.-L., Yu, Z.-Y., Wang, J.-T., Zhang, X.-M., Zhang, Q., Wang, N.-N., Huang, Y.-Y., Zhou, H.-G., and Guo, J.-C. (2021). Front. Pharmacol. 12:754387. doi: 10.3389/ fphar.2021.754387

In the published article, there was an error in affiliation 1 and 4 . Instead of "1 Department of Geriatrics of Huashan Hospital, National Clinical Research Center for Aging and Medicine, Fudan University, Shanghai, China" and "4 Department of Translational Neuroscience, Jing'an District Centre Hospital of Shanghai and State Key Laboratory of Medical Neurobiology and MOE Frontiers Center for Brain Science, Institutes of Brain Science, Fudan University, Shanghai, China," it should be ${ }^{1}$ Department of Geriatrics of Huashan Hospital, National Clinical Research Center for Aging and Medicine, Department of Translational Neuroscience, Jing'an District Centre Hospital of Shanghai, State Key Laboratory of Medical Neurobiology and MOE Frontiers Center for Brain Science, Institutes of Brain Science, Fudan University, Shanghai, China."

The authors apologize for this error and state that this does not change the scientific conclusions of the article in any way. The original article has been updated.

Publisher's Note: All claims expressed in this article are solely those of the authors and do not necessarily represent those of their affiliated organizations, or those of the publisher, the editors and the reviewers. Any product that may be evaluated in this article, or claim that may be made by its manufacturer, is not guaranteed or endorsed by the publisher.

Copyright () 2022 Wang, Mi, Jin, Guo, Yu, Wang, Zhang, Zhang, Wang, Huang, Zhou and Guo. This is an open-access article distributed under the terms of the Creative Commons Attribution License (CC BY). The use, distribution or reproduction in other forums is permitted, provided the original author(s) and the copyright owner(s) are credited and that the original publication in this journal is cited, in accordance with accepted academic practice. No use, distribution or reproduction is permitted which does not comply with these terms. 